\title{
The British Journal of Psychiatry: Achieving Excellence
}

\section{GREG WILKINSON}

Our aim is to consolidate the position of the British Journal of Psychiatry as the leading international journal of general psychiatry and an essential companion in clinical practice. We seek to do this in two ways: by ensuring rapid publication of the best clinical science and art; and by enhancing our editorial standards and practices. All articles submitted to the $B J P$ are subject to peer review.

Innovations will be designed to inform, educate, stimulate and, occasionally, entertain readers. Editorials, beginning this month, will be topical, authoritative, but personal and thought-provoking views on current themes. We will introduce a peerreview section in which an article will appear with assessments and author's further response. We intend to broaden our appeal to readers by widening our selection of articles.

The $B J P$ now publishes the dates of submission, final revision and acceptance of articles to show the duration of the peer-review process and to indicate where delays occur. Authors can expect to receive a decision on acceptance of their article, or otherwise, within three months of submission. Our target is to respond to $80 \%$ of authors within two months. We invite authors to return revisions within one month. Our target is to publish $90 \%$ of the material subject to peer review within six months of acceptance. We will report the results of our target setting annually.

Authors can choose to have their work peer reviewed anonymously. Those choosing this option must submit their work without personal identification. They must provide the names and addresses of all authors along with a covering letter to the Editor.

From 1 July 1994 the BJP will publish structured summaries. All articles submitted from now onwards should include a summary of up to 150 words, comprising four sections: background, method, results, and conclusions. These should outline the questions addressed, the design, essential findings, and main conclusion of the study.

The scientific quality of the $B J P$ depends largely on peer review and the valuable contributions made by our panel of assessors. We are improving our service to them by providing copies of co-assessors' reports and informing them about the fate of papers they have assessed. We are expanding the number of assessors, particularly in the field of clinical psychiatry and its specialties. We are also expanding the numbers of Assistant and Corresponding Editors, each of whom will have a three-year tenure. Assessors and editors have a key role in advancing our overriding concern, which is to improve the prevention, investigation, diagnosis, treatment, and care of mental illness, and the promotion of mental health, throughout the world.

The Royal College, in addition to publishing the $B J P$, will continue to serve psychiatrists and allied professionals through its book programme under the Gaskell imprint. A major College Seminar Series aimed at psychiatrists in training is being published. Another crucial area of development is the introduction of a bi-monthly continuing medical education periodical, provisionally entitled Advances in Psychiatric Treatment, edited by Professor Andrew Sims, and due for publication in July 1994.

The Psychiatric Bulletin, which began life 23 years ago as a College noticeboard in this journal, is being relaunched this month under the editorship of Dr Alan Kerr, with the subtitle Journal of Trends in Psychiatric Practice, and a new design to reflect the changes which have led to its position as the journal of developments in psychiatric practices and service provision.

The $B J P$ has a British base and an international perspective. We will encourage the involvement, and commitment, of readers, authors, assessors, and editors. We will welcome constructive criticism, and will adapt to changing needs and technological developments. In essence: we will strive for excellence in clinical communication.

Greg Wilkinson,

Editor, The British Journal of Psychiatry

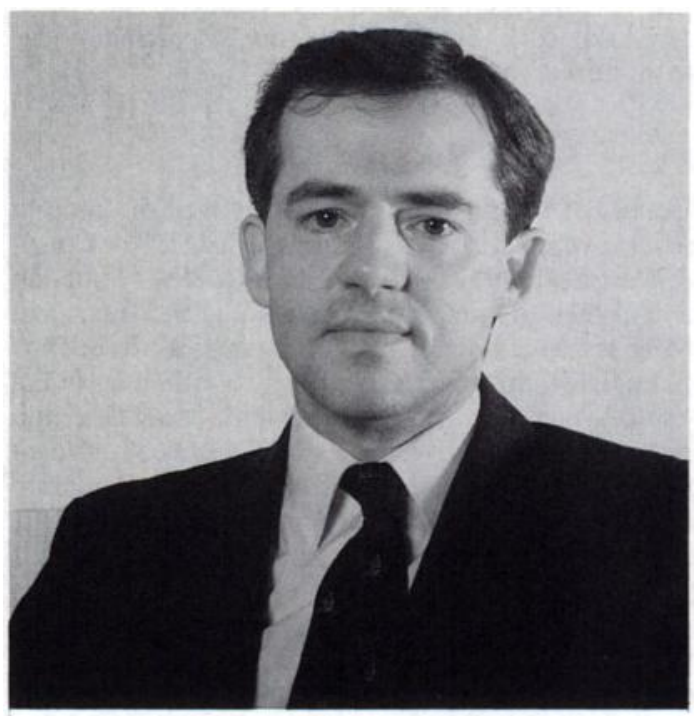

